\title{
Symbolic-Numerical Modeling of the Influence of Damping Moments on Satellite Dynamics
}

\author{
Sergey A. Gutnik ${ }^{1,2, \star}$ and Vasily A. Sarychev ${ }^{3, \star \star}$ \\ ${ }^{1}$ Moscow State Institute of International Relations 76, Prospekt Vernadskogo, Moscow, 119454, Russia \\ ${ }^{2}$ Moscow Institute of Physics and Technology, 9 Institutskiy lane, Dolgoprudny, 141700, Russia \\ ${ }^{3}$ Keldysh Institute of Applied Mathematics (RAS) 4, Miusskaya Square, Moscow, 125047, Russia
}

\begin{abstract}
The dynamics of a satellite on a circular orbit under the influence of gravitational and active damping torques, which are proportional to the projections of the angular velocity of the satellite, is investigated. Computer algebra Gröbner basis methods for the determination of all equilibrium orientations of the satellite in the orbital coordinate system with given damping torque and given principal central moments of inertia were used. The conditions of the equilibria existence depending on three damping parameters were obtained from the analysis of the real roots of the algebraic equations spanned by the constructed Gröbner basis. Conditions of asymptotic stability of the satellite equilibria and the transition decay processes of the spatial oscillations of the satellite at different damping parameters have also been obtained.
\end{abstract}

\section{Introduction}

In this paper, a symbolic-numerical study of the satellite dynamics under the influence of gravitational and active damping torques is presented. The gravity oriented satellite with different moments of inertia in the central Newtonian force field in a circular orbit has 24 equilibrium orientations and four of them are stable [1]. An important property of the gravity orientation systems is that these systems can operate for a long time without needing fuel spending. In the present work we analyze the behavior of a satellite acted upon by the gravity gradient and active damping torques. The action of damping torques can provide the asymptotic stability of the equilibria of the gravity oriented satellites. Therefore, it is important to investigate the joint action of the gravitational and active damping torques and to analyze the necessary and sufficient conditions for asymptotic stability of the satellite equilibria on a circular orbit.

In the present work, the problem of determination of the classes of equilibrium orientations and the conditions for asymptotic stability of defined equilibria for the general values of damping torques is considered. The investigation of equilibria was performed by application of the Computer Algebra Gröbner basis methods. The types of transition decay processes of spatial oscillations of the satellite at different damping parameters have been investigated numerically.

The computer algebra methods for the investigation of the asymptotic stability of the equilibrium orientations of a satellite were successfully used earlier for another model of damping torques [2].

${ }^{\star}$ e-mail: s.gutnik@inno.mgimo.ru

$\star \star$ e-mail: vas31@ rambler.ru 


\section{Equations of Motion}

Consider the motion of the satellite-rigid body subjected to gravitational and active damping torques on a circular orbit. We assume that the active damping torques are proportional to the projections of the angular velocity of the satellite. To write the equations of motion we introduce two right-handed Cartesian coordinate systems with origin at the satellite center of mass $O$. The orbital coordinate system $O X Y Z$; the axis $O Z$ is directed along the radius-vector from the Earth center of mass and the satellite center of mass; the axis $O X$ is along the direction of the satellite orbital motion. The satellite body coordinate system $O x y z ; O x, O y$, and $O z$ are the principal central axes of inertia of the satellite. The orientation of the satellite body coordinate system $O x y z$ with respect to the orbital coordinate system is determined by means of the aircraft angles of pitch $(\alpha)$, yaw $(\beta)$, and roll $(\gamma)$. The direction cosines of the transformation matrix between the orbital coordinate system $O X Y Z$ and $O x y z$ are given in [3].

Let the satellite be acted upon by the moments of active damping, their integral vector projections on the axis $O x, O y$, and $O z$ are equal respectively to: $M_{x}=\bar{k}_{1} p_{1}, M_{y}=\bar{k}_{2} q_{1}$, and $M_{z}=\bar{k}_{3} r_{1}$. Here $\bar{k}_{1}, \bar{k}_{2}$, and $\bar{k}_{3}$ are the damping coefficients, $p_{1}, q_{1}$, and $r_{1}$ are the projections of the satellite angular velocity onto the axes $O x, O y$, and $O z ; \omega_{0}$ is the angular velocity of the orbital motion of the satellite center of mass. Then, the equations of satellite attitude motion can be written in the dimensionless Euler form:

$$
\begin{array}{r}
\theta_{A} \dot{p}+\left(\theta_{C}-1\right) q r-3\left(\theta_{C}-1\right) a_{32} a_{33}+\tilde{k}_{1} p=0, \\
\dot{q}+\left(\theta_{A}-\theta_{C}\right) r p-3\left(\theta_{A}-\theta_{C}\right) a_{31} a_{33}+\tilde{k}_{2} q=0, \\
\theta_{C} \dot{r}+\left(1-\theta_{A}\right) p q-3\left(1-\theta_{A}\right) a_{31} a_{32}+\tilde{k}_{3} r=0, \\
p=(\dot{\alpha}+1) a_{21}+\dot{\gamma}, \quad q=(\dot{\alpha}+1) a_{22}+\dot{\beta} \sin \gamma, \quad r=(\dot{\alpha}+1) a_{23}+\dot{\beta} \cos \gamma .
\end{array}
$$

Here, $\theta_{A}=A / B, \theta_{C}=C / B$ denote dimensionless inertial parameters, $A, B$, and $C$ are the principal central moments of inertia of the satellite, $p=p_{1} / \omega_{0}, q=q_{1} / \omega_{0}, r=r_{1} / \omega_{0}, \tilde{k}_{1}=\bar{k}_{1} / B \omega_{0}$, $\tilde{k}_{2}=\bar{k}_{2} / B \omega_{0}, \tilde{k}_{3}=\bar{k}_{3} / B \omega_{0}, \tau=\omega_{0} t$. The dot denotes differentiation with respect to $\tau$.

\section{Equilibrium Orientations of the Satellite}

Putting in (1) $\alpha=\alpha_{0}=$ const, $\beta=\beta_{0}=$ const, $\gamma=\gamma_{0}=$ const, we obtain the stationary equations

$$
a_{22} a_{23}-3 a_{32} a_{33}+k_{1} a_{21}=0, a_{21} a_{23}-3 a_{31} a_{33}+k_{2} a_{22}=0, a_{21} a_{22}-3 a_{31} a_{32}+k_{3} a_{23}=0,
$$

which specifies the equilibrium orientations of the satellite in the orbital coordinate system at $A \neq B \neq C$, such that the orthogonality conditions for the direction cosines are fulfilled

$$
a_{i 1} a_{j 1}+a_{i 2} a_{j 2}+a_{i 3} a_{j 3}-\delta_{i j}=0,
$$

where $\delta_{i j}$ is the Kronecker delta and $(i, j=1,2,3)$. Here $k_{1}=\tilde{k}_{1} /(C-B), k_{2}=\tilde{k}_{2} /(A-C)$, $k_{3}=\tilde{k}_{3} /(B-A)$. We will consider the case when the damping coefficients $k_{1}, k_{2}$ and $k_{3}$ are positive.

Equations (2) and (3) form a closed system of equations with respect to the six direction cosines identifying the satellite equilibrium orientations. For this system of equations we formulate the following problem: given $k_{1}, k_{2}$, and $k_{3}$, determine all the nine directional cosines, i.e., all satellite equilibrium orientations in the orbital coordinate system. After $a_{21}, a_{22}, a_{23}, a_{31}, a_{32}$, and $a_{33}$ are found, the direction cosines $a_{11}, a_{12}$ and $a_{13}$ can be determined from the conditions of orthogonality.

To find solutions of the algebraic system (2)-(3) we used the algorithm for constructing the Gröbner bases [4], implemented as the package Groebner [Basis] in the computer algebra system Maple 
15 [5]. We constructed the Gröbner basis of the system of six second-order polynomials (2)-(3) with six variables $a_{i j}(i=2,3 ; j=1,2,3)$, with respect to the lexicographic ordering of variables by using the option plex. The list of polynomials $F$ includes the left-hand sides of algebraic equations. (2), (3): G:=map (factor, Groebner [Basis] (F, plex (a31, .. a22))). Below we write down the polynomial in the Gröbner basis that depends only on one variable $x=a_{22}$. This polynomial has the simple form

$$
a_{22}\left(a_{22}^{2}-1\right)=0 \text {. }
$$

The polynomials from the Gröbner basis that depend on the variables $a_{21}, a_{23}$ have similar forms $\left(a_{21}\left(a_{21}^{2}-1\right)=0, a_{23}\left(a_{23}^{2}-1\right)=0\right)$. The polynomial from the Gröbner basis that depends on the variable $a_{33}$ is: $a_{33}\left(a_{33}^{4}-9 a_{33}^{2}+k_{2}^{2}\right)\left(\left(a_{33}^{4}-9 a_{33}^{2}+k_{1}^{2}\right)=0\right.$.

To determine all equilibria, it is required to consider separately the following three cases: $a_{22}=1$, $a_{22}=-1$, and $a_{22}=0$. In the first and second cases, i.e., $a_{22}= \pm 1\left(a_{21}=a_{23}=0\right)$ the system (2), (3) results in the following equilibrium solutions $\left(k_{2}<9 / 4\right)$ :

$$
a_{22}= \pm 1, a_{21}=a_{22}=a_{32}=0 ; a_{31}^{2}=\left(3 \mp \sqrt{9-4 k_{2}^{2}}\right) / 6, a_{33}^{2}=\left(3 \pm \sqrt{9-4 k_{2}^{2}}\right) / 6 .
$$

In the third case, $a_{22}=0$, it is easy to find from the system (2)-(3) two sets of simple equilibrium solutions.

\section{Necessary and Sufficient Conditions of Asymptotic Stability of the Equilibrium Orientations of a Satellite}

In order to study the necessary and sufficient conditions of asymptotic stability of the determined above equilibrium orientations of the system (2)-(3) we use the linearized system of equations (1) in the vicinity of the equilibrium solution (5)

$$
\alpha_{0}= \pm \arcsin \left(\left[\left(3-\sqrt{9-4 k^{2}}\right) / 6\right]^{1 / 2}\right), \quad \beta_{0}=\gamma_{0}=0 .
$$

The quantities $\alpha, \beta$, and $\gamma$ are written in the form $\alpha=\alpha_{0}+\bar{\alpha}, \beta=\beta_{0}+\bar{\beta}, \gamma=\gamma_{0}+\bar{\gamma}$, where $\bar{\alpha}, \bar{\beta}$ and $\bar{\gamma}$ are small deviations from the equilibrium orientation of the satellite $\alpha=\alpha_{0}, \beta=\beta_{0}, \gamma=\gamma_{0}$, satisfying the system of equations (2)-(3).

Now let us consider the small oscillations of the satellite in the vicinity of the specific equilibrium orientation (6) in the case $k_{1}=k_{2}=k_{3}=k$. The linearized equations in this case take the form

$$
\begin{array}{r}
\ddot{\bar{\alpha}}+k \dot{\bar{\alpha}}+3\left(\theta_{A}-\theta_{C}\right)\left(\cos ^{2} \alpha_{0}-\sin ^{2} \alpha_{0}\right) \bar{\alpha}=0, \\
\theta_{C} \ddot{\bar{\beta}}+k \dot{\bar{\beta}}-\left(\theta_{A}+\theta_{C}-1\right) \dot{\bar{\gamma}}+\left(1-\theta_{A}\right)\left(1+3 \sin ^{2} \alpha_{0}\right) \bar{\beta}+\left[3\left(1-\theta_{A}\right) \sin \alpha_{0} \cos \alpha_{0}-k\right] \bar{\gamma}=0, \\
\theta_{A} \ddot{\ddot{\gamma}}+k \dot{\bar{\gamma}}+\left(\theta_{A}+\theta_{C}-1\right) \overline{\bar{\beta}}+\left(1-\theta_{C}\right)\left(3 \cos ^{2} \alpha_{0}+1\right) \bar{\gamma}+\left[3\left(1-\theta_{C}\right) \sin \alpha_{0} \cos \alpha_{0}+k\right] \bar{\beta}=0 .
\end{array}
$$

The characteristic equation of the system (7)

$$
\left[\lambda^{2}+k \lambda+3\left(\theta_{A}-\theta_{C}\right) \sqrt{9-4 k^{2}}\right]\left(A_{0} \lambda^{4}+A_{1} \lambda^{3}+A_{2} \lambda^{2}+A_{3} \lambda+A_{4}\right)=0 .
$$

decomposes into quadratic and 4th degree equations. Here, the following notations are used:

$$
\begin{aligned}
& A_{0}=\theta_{A} \theta_{C}, \quad A_{1}=k\left(\theta_{C}+\theta_{A}\right), \quad A_{4}=\left(1-\theta_{A}\right)\left(1-\theta_{C}\right)\left(3+k^{2}\right)+\left(\theta_{A}+\theta_{C}-1\right) k^{2} . \\
& A_{2}=k^{2}+\left(\theta_{A}+\theta_{C}-1\right)^{2}+\left[\theta_{A}\left(1-\theta_{A}\right)\left(5-\sqrt{9-4 k^{2}}\right)+\theta_{C}\left(1-\theta_{C}\right)\left(5+\sqrt{9-4 k^{2}}\right)\right] / 2, \\
& A_{3}=k\left[\left(\theta_{A}+\theta_{C}-1\right)\left(\theta_{A}-\theta_{C}+2\right)+\left[\left(1-\theta_{C}\right)\left(5+\sqrt{9-4 k^{2}}\right)+\left(1-\theta_{A}\right)\left(5-\sqrt{9-4 k^{2}}\right)\right] / 2\right] .
\end{aligned}
$$


The necessary and sufficient conditions for the asymptotic stability (Routh-Hurwitz criterion) of the equilibrium solution (6) take the following form:

$$
\begin{gathered}
k>0, \theta_{A}-\theta_{C}>0, \Delta_{1}=A_{1}>0, \Delta_{2}=A_{1} A_{2}-A_{0} A_{3}>0, \\
\Delta_{3}=A_{1} A_{2} A_{3}-A_{0} A_{3}^{2}-A_{1}^{2} A_{4}>0, \Delta_{4}=\Delta_{3} A_{4}>0,\left(A_{4}>0\right) .
\end{gathered}
$$

The detailed analysis of the regions where necessary and sufficient conditions of stability hold was studied in the plane of the two dimensionless inertia parameters $\left(\theta_{A}, \theta_{C}\right)$ at different values of the damping coefficient $k$. It is evident that along with the conditions of stability the triangle inequalities for a rigid body should also be satisfied, $\theta_{A}+\theta_{C}>1, \theta_{C}+1>\theta_{A}, \theta_{A}+1>\theta_{C}$. One may disregard the third triangle inequality, since it holds automatically at $\theta_{A}>\theta_{C}$. Thus, the region is limited by the straight lines $\theta_{C}=1-\theta_{A}, \quad \theta_{C}=\theta_{A}, \quad \theta_{C}=\theta_{A}-1$.

An example of such region and also all the lines at which the inequalities convert into equalities for $k=0.5$ is shown in Figure 1 left. The region where the necessary and sufficient conditions of stability are satisfied is bounded by the above defined straight lines and by the hyperbola $\Delta_{4}=0$ and are marked out by black color.

The numerical integration of system (1) has been done for different values of the parameter $k$. The different types of transition decay processes of the spatial oscillations of the satellite at different damping parameters has been investigated numerically. Figure 1 right shows an example of transition decay processes of spatial oscillations for $k=0.5$ at inertia parameters $\theta_{A}=0.9, \theta_{C}=0.2$, where the conditions of asymptotic stability hold. The system in this case reaches the equilibrium position (6) at all three angles, at the $\tau$ value equal to 25 , which correspond to the 4 satellite turnover in the orbit.
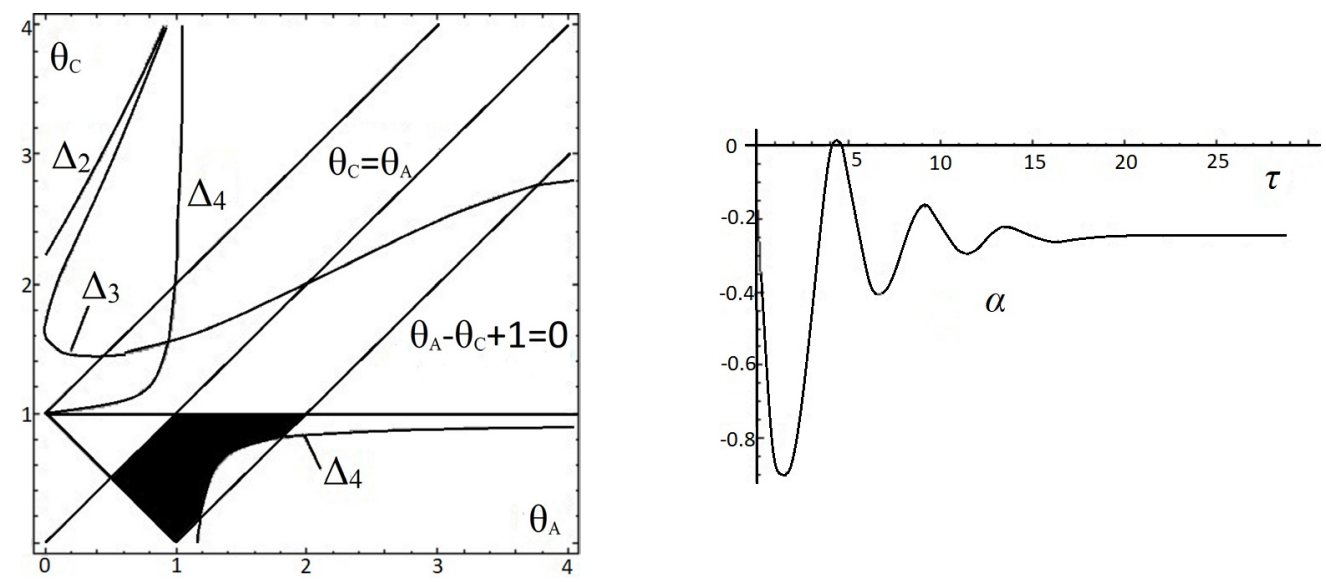

Figure 1. Left: The region of fulfillment of the asymptotic stability conditions for $k=0.5$. Right: The transitional process of damping oscillations for $k=0.5, \alpha_{0}=-0.2$

\section{References}

[1] Beletsky V.V., Motion of Satellite in Gravitational Field (MGU Press, Moscow, 1975) 416

[2] Gutnik, S.A., Sarychev, V.A., LNCS 10490, 167-182 (2017)

[3] Sarychev V.A., Problems of orientation of satellites. Itogi Nauki i Tekhniki. Ser. "Space Research" 11, (VINITI , Moscow, 1978) 222

[4] Buchberger B., SIGSAM Bulletin 3, 19-29 (1976)

[5] Maple online help (http://maplesoft.com/support/help/) 